\title{
Ausbildung und berufliche Qualifikation von Erwachsenen mit Myotonen Dystrophien - eine fehlgeleitete Wahrneh- mung durch die Facies myopathica?
}

\section{Educational and Professional Qualifications of Adults With Myotonic Dystrophies - A Misleading Perception by the Myopathic Face?}

Autoren

Institut
K. Stahl, S. Wenninger, A. Schüller, F. Montagnese, B. Schoser

Interdisziplinäres Zentrum für Neuromuskuläre Erkrankungen des Klinikums der Universität München, Ludwig-MaximiliansUniversität München, Friedrich-Baur-Institut, Neurologische Klinik, München

\section{Schlüsselwörter \\ - Myotone Dystrophie \\ - DM1 \\ DM2 \\ - Bildungsniveau \\ - Intelligenzminderung \\ Key words \\ - myotonic dystrophy \\ - DM1 \\ - DM2 \\ - educational level \\ - mental retardation}

\section{Bibliografie}

Dol http://dx.doi.org/ 10.1055/s-0042-104193

Fortschr Neurol Psychiatr 2016;

84: 211-216 @ Georg Thieme

Verlag KG Stuttgart · New York

ISSN 0720-4299

Korrespondenzadresse

Professor Dr. med. Benedikt

Schoser

Friedrich-Baur-Institut, Neurologische Klinik, Interdisziplinäres Zentrum für Neuromuskuläre Erkrankungen des Klinikums der Universität München, Ludwig-MaximiliansUniversität München

Ziemssenstr. 1a

D-80336 München

Tel.: ++ 49/89/4400 57400

bschoser@med.uni-

muenchen.de

\section{Zusammenfassung \\ $\nabla$}

Hintergrund: Myotone Dystrophien Typ 1 und 2 (DM1 / DM2) sind die häufigsten progressiven, segmental progeroiden, multisystemischen, erblichen Muskelerkrankungen im Erwachsenenalter. Die Störung der Exekutivfunktionen bei DM1-Patienten stellt einen wesentlichen Krankheitsanteil dar. Durch die pathognomonische Facies myopathica wird u.a. ein niedriges Bildungsniveau bei DM1 suggeriert. Detailliertere Untersuchungen zum Bildungsniveau dieser Patientengruppen existieren aber nicht.

Methoden: Wir nutzten einen standardisierten strukturierten Fragebogen zur Untersuchung des Bildungsniveaus bei Patienten mit molekulargenetisch gesicherter DM1 und DM2. Inhalte der Untersuchung umfassten das Bildungsniveau, den höchsten erreichten Schulabschluss und die berufliche Qualifikation. Die erhobenen Daten wurden mit vorliegenden Daten zur Bildung der Normalbevölkerung verglichen.

Ergebnisse: Aus einem Gesamtkollektiv von 546 DMPatienten nahmen 125 DM1- und 156 DM2Patienten (51\%) an dieser Studie teil. Für den höchsten Schulabschluss bestand ein statistisch signifikanter Unterschied zwischen den Kollektiven und der Normalbevölkerung. 50,4\% der DM1und 48,3\% der DM2-Patienten erreichten die (Fach-)Hochschulreife, während es in der Normalbevölkerung durchschnittlich $29,6 \%$ sind. Für die weitere berufliche Qualifizierung fanden sich nur geringe Abweichungen zwischen den Kohorten (Fach-/Hochschulabschluss: DM1: 25\%, DM2 23\%; Ausbildung/Lehre: DM1: 61\%, DM2 71\%) und der Normalbevölkerung. Es zeigte sich eine signifikant erhöhte Prävalenz von „Rechtschreibproblemen“ $(p=0,039)$, „Schwierigkeiten beim Kopfrechnen“ $(p=0,043)$, und Einstufung der Patienten „mit Lernproblemen“ ( $p=0,012)$ bei DM1-Patienten.

Diskussion: Die allgemeine klinische Wahrnehmung der DM1-Patienten ist u. a. durch die Facies

\section{Abstract \\ $\nabla$}

Background: Myotonic dystrophies types 1 and 2 (DM1 / DM2) are the most frequent inherited progressive, segmental progeroid, multisystemic neuromuscular diseases in adulthood. The executive impairment is one of the key disease features. The myopathic face triggers the general perception of DM1 patients being associated with a low educational level.

Methods: We used a standardized questionnaire to evaluate educational levels in adults with genetically confirmed DM1 and DM2 in comparison to data of the general population. Investigated topics included the level of education, e. g. the highest university degree aquired.

Results: Out of a total cohort of 546 DM patients, 125 DM1 and 156 DM2 patients (51\%) participated in this study. There was no statistically significant difference between the two collectives as far as high school levels are concerned. $50.4 \%$ of DM1 and $48.3 \%$ of DM2 patients obtained the higher education entrance qualification compared to $29.6 \%$ of the normal German population. However, there were significant differences between the two collectives in "spelling problems" (DM1 cohort: $\mathrm{p}=0.039$ ), "difficulty in mental arithmetic" $(p=0.043)$, and classification of patients "with learning difficulties" ( $p=0.012$ ).

Discussion: Misled by a myopathic face, many physicians associate myotonic dystrophy with cognitive deficiency. Based on our study, the minimal deviation between DM1 and DM2 and the normal German population indicates that the multisystemic disease does not significantly influence the maximum attainable level of education in adults with DM1.

Conclusion: In summary, physicians should be aware that the general educational levels are rather normal in patients with myotonic dystrophy type 1 and rethink their perception of DM1 patients. 
myopathica determiniert. Mit der Schwäche der Gesichtsmuskulatur und dem offenstehenden Mund wird ein kognitives Defizit assoziiert, das diese Patientengruppe stigmatisiert. Die in unserer Studie dargestellten minimalen Abweichungen zwischen den Patientenkollektiven und der Normalbevölkerung weisen darauf hin, dass das erreichbare Bildungsniveau durch die multisystemische Erkrankung nicht wesentlich eingeschränkt wird.

Beurteilung: Die im klinischen Alltag vorhandene Fehleinschätzung der kognitiven Leistungsfähigkeit von Erwachsenen mit Myotonen Dystrophien sollte revidiert werden.

\section{Einleitung}

Die Myotonen Dystrophien sind ein typisches Beispiel der segmentalen progeroiden Erkrankungen. Unimodale progeroide Erkrankungen weisen Manifestationen an einem einzelnen Organ auf, während die sog. segmentalen progeroiden Syndrome multiple Organmanifestationen zeigen [1]. Ein segmentales progeroides Syndrom zeigt ein paralleles Auftreten vorzeitiger Alterung in multiplen Organen wie der Haut, dem Skelett, der Muskulatur, dem Stoffwechsel mit Diabetes mellitus, auffälliger Atrophie des subkutanen Fettgewebes, neurodegenerativer Erkrankungen, erhöhter Inzidenz unterschiedlicher Tumoren und gehäuften Auftretens von Autoimmunerkrankungen. Ein weiteres Merkmal progeroider Syndrome ist, dass eine ursächliche Keimbahnmutation alle Organe betrifft, aber diese Mutation in sog. postmitotischen Organen wie der Muskulatur und dem Nervensystem andere klinische Auswirkungen als für mitotisch aktive Gewebe wie Bindegewebe, epithelial aufgebaute Organe oder das Blutsystem hat [2]. Alle diese Merkmale treffen auf die Myotonen Dystrophien zu.

In der namensgebenden Erstbeschreibung der Myotonen Dystrophie Typ 1 (DM1) von Hans Steinert aus dem Jahr 1909 standen die klassischen muskulären Symptome wie Muskelatrophie und Myotonie ganz im Vordergrund [3]. Bereits 1923 wurde durch Adie und Greenfield [4], sowie später in zahlreichen Fallberichten und Studien, das kognitive Defizit dieser Patientengruppe als Teil der multisystemischen Erkrankung beschrieben [5-7]. Die ab 2001 mögliche genetische Unterscheidung der Myotonen Dystrophien in zwei Formen (DM1 und DM2) ermöglichte es zudem, die neuropsychologische Komponente der DM1 eindeutiger zu charakterisieren [8]. Molekulargenetisch liegt bei beiden Formen eine abnorme Repeat-Verlängerung im entsprechenden Genlokus vor. Bei der DM1 liegt eine Expansion des CTG-Triplets von mehr als 50 Kopien im Myotonin-Protein-Kinase-Gen (DMPK) vor, bei der DM2 eine CCTG-Repeat-Verlängerung von mehr als 75 Kopien im Zinkfinger-9-Gen (ZNF9) [8-10], die sich u.a. in Form einer sog. „Spliceopathy“ äußern. Nach derzeitigem Kenntnisstand stellen Unterschiede in der Repeatlänge sowie andere noch unbekannte Mechanismen für Patienten mit DM1 hinsichtlich Erkrankungsbeginn und Symptomausprägung ein wichtiges Kriterium dar [11]. Neben der adulten Form mit Symptombeginn im Erwachsenenalter und der davon nur unscharf abgrenzbaren juvenilen Form existiert eine schwer verlaufende kongenitale Form, die bereits intrauterin bzw. postpartal beginnt [10].

Frühere Untersuchungen beschrieben oft allgemein ein „kognitives Defizit" bei den DMs [4-6]. Aktuelle Untersuchungen differenzieren nun diese neuropsychologischen Einschränkungen, die von geringen Konzentrationsstörungen über Tagesmüdigkeit bis hin zu schwerer Fatigue-Symptomatik oder Autismus-Spektrums-Erkrankungen reichen können. Für einen Großteil der
DM1-Patienten stehen diese neuropsychologischen Symptome oftmals mehr im Vordergrund als die muskuläre Komponente [12-14]. Diese beeinflussen den Tagesrhythmus der Patienten deutlich, erschweren den beruflichen Alltag und führen nicht selten zu frühzeitiger Erwerbsminderung und sozialem Rückzug. Im klinischen Alltag werden Patienten mit DM1 durch ihr klinisches Stigma einer eindrücklichen Gesichtsmuskulaturschwäche mit offenstehendem Mund, der typischen Facies myopathica ( $\bullet$ Abb. 1), und der frontalen Glatzenbildung als allgemein bildungsfern und intelligenzgemindert eingestuft [6]. Diese sichtbare Veränderung des Gesichts liegt im Verlauf der DM1-Erkrankung bei nahezu allen Betroffenen vor. In der Summe werden das Sozialleben und der Alltag der Patienten von dieser Stigmatisierung enorm beeinflusst. Ein sozialer Rückzug ist sehr häufig anzutreffen. Unsere Untersuchung zeigt nun, dass das resultierende Bildungsniveau sowie die berufliche Laufbahn eines Großteils der DM-Patienten von der kognitiven Erkrankung viel weni-

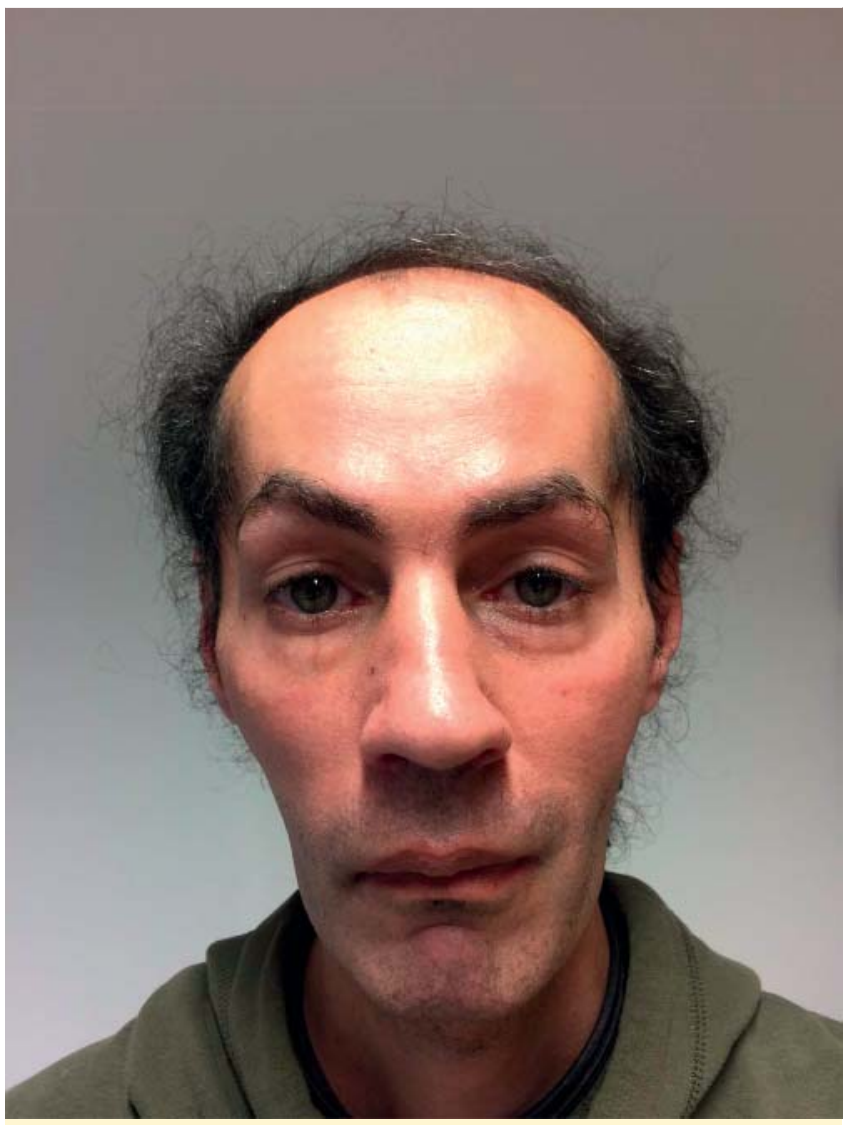

Abb. 1 DM1-Patient mit Facies myopathica, Stirnglatze und Temporalisatrophie. 
ger beeinträchtigt sind als allgemein angenommen. Die kognitive Fehleinschätzung des Klinikers, getriggert durch die stigmatisierende Facies myopathica, ist häufig. Diese Fehlwahrnehmung und daraus folgende Fehlleitungen in der Betreuung sollten langfristig geändert werden, um diesen Patienten in der klinischen Versorgung und Beratung gerechter zu werden. Ziel unserer Studie war es, das Leistungsniveau von Patienten mit Myotonen Dystrophien anhand von Daten zur schulischen und beruflichen Entwicklung im Vergleich zu Daten der Normalbevölkerung zu evaluieren.

\section{Patienten und Methoden \\ $\nabla$}

Ein Gesamtkollektiv von 546 DM Patienten wurde für diese Studie aufgefordert teilzunehmen. Das untersuchte Studienkollektiv (51\% des Gesamtkollektivs) setzt sich aus 125 DM1-Patienten mit vorwiegend adulter Form und 156 DM2-Patienten zusammen, die in der Datenbank des Friedrich-Baur-Instituts oder dem DM-Patientenregister für Deutschland gemeldet sind. Einschlusskriterium für die Untersuchung war ein positiver genetischer Befund für DM1 oder DM2. Zusätzliche Grundlage für die Datenerhebung war ein validierter strukturierter „Dyslexia“-Fragebogen der Liverpool John Moores University [15]. Dessen Inhalte umfassen dabei Fragen zur schulischen Laufbahn wie Schulwechsel, höchster Schulabschluss und Abschlussnoten. Ein Fragenblock untersucht Lernschwierigkeiten während der gesamten Schulzeit und enthält die Bereiche „Lesen lernen“, „Rechtschreibung erlernen“, „Schreiben lernen“, „Kopfrechnen“ sowie die allgemeine Einstufung „mit Lernproblemen“. Daneben werden die berufliche Entwicklung mit Ausbildung und Studium sowie Lese-Rechtschreibprobleme erfasst. Alle Daten wurden mittels deskriptiver und explorativer Analytik und statistischer Tests (Shapiro-Wilks-Test bzw. Kolmogorov-Smirnov, Chi-Quadrat-Test nach Pearson) untersucht (IBM SPSS Statistics 23.0). Alle beschriebenen Untersuchungen am Menschen wurden mit Zustimmung der zuständigen Ethik-Kommission, im Einklang mit nationalem Recht sowie gemäß der Deklaration von Helsinki von 1975 (in der aktuell überarbeiteten Fassung) durchgeführt. Von allen beteiligten Patienten liegt eine schriftliche Einverständniserklärung vor. Alle Patienten, die über Bildmaterial oder anderweitige Angaben innerhalb des Manuskripts zu identifizieren sind, haben dazu ihre schriftliche Einwilligung gegeben.

\section{Ergebnisse \\ $\nabla$}

Von 546 versandten Fragebögen wurden 281 (51\%) zurückgesandt (125 DM1, 156 DM2). Das Alter war in beiden Patientenkollektiven normalverteilt. Das Durchschnittsalter für Patienten der DM1-Gruppe mit 47,2\% Frauen betrug 43,73 $\pm 1,18$ Jahre (10 - 77 Jahre) und für Patienten der DM2-Gruppe mit 66\% Frauen 54,86 $\pm 0,98$ Jahre (14-82 Jahre). 3,2\% der Patienten weisen eine kongenitale DM1-Form auf. Entsprechend vorliegenden Daten beträgt die Repeatlänge der DM1-Patienten im Durchschnitt $508 \pm 351$ CTG-Repeats (70-1550; Median 425). Im Bereich <300 CTG-Repeats liegen 28 DM1-Patienten (29,2\%), 42 Patienten $(43,8 \%)$ zwischen 300 und 600 CTG-Repeats, 13 (13,5\%) zwischen 601 und 900 und weitere 13 Patienten (13,5\%) weisen eine Repeat-Verlängerung >900 auf. Bei 29 Patienten erfolgte die Diagnosesicherung über die Familie oder die Muskelbiopsie. Der Erkrankungsbeginn konnte bei 92 DM1-Patienten ermittelt werden und fällt durchschnittlich in ein Alter von 24,24 \pm 1,41 Jahren
(1 - 54 Jahre). Bei 82 DM2-Patienten liegt der Erkrankungsbeginn im Durchschnitt bei 40,38 $\pm 1,55$ Jahren ( 7 - 68 Jahre).

Schulabschluss: 92,0\% des DM1- und 94,2\% des DM2-Kollektivs erzielten einen allgemeinen Schulabschluss. Davon erlangten die (Fach-)Hochschulreife auf dem Gymnasium bzw. an der Fachoberschule/Berufsoberschule 50,4\% der DM1- und 48,3\% der DM2-Patienten. In der DM1-Gruppe ( $=115)$ erreichten $26,1 \%$ die mittlere Reife und 20,9\% einen Hauptschulabschluss. Von 147 DM2-Patienten gaben 31,3\% die mittlere Reife und 17,7\% den Hauptschulabschluss als höchsten Schulabschluss an. Noch in schulischer Ausbildung befinden sich 2,4\% DM1- und 0,6\% DM2-Patienten. Keine Angaben zum Schulabschluss machten 5,6\% der DM1- bzw. 5,1\% der DM2-Patienten an ( 0 Tab.1). Schulwechsel: Bei 49,0\% der DM1- und 31,3\% DM2-Patienten fand ein Schulwechsel statt (Grundschule ausgeschlossen). Davon sind $86,3 \%(84,8 \%$ DM2) schulisch aufgestiegen und 19,6\% (10,9\% DM2) abgestiegen.

Lernschwierigkeiten: Die dichotom gestellten Fragen zu Lernschwierigkeiten wurden mit „ja“ beantwortet bei: „Erlernen des Lesens“ 17,6\% DM1 (17,3\% DM2), „Rechtschreibung erlernen“ 32,0\% DM1 (21,2\% DM2), „Schreiben lernen“ 12,0\% DM1 (12,2\% DM2), „Probleme beim Kopfrechnen“ 29,6\% DM1 (19,2\% DM2) und Einstufung der Patienten „mit Lernproblemen“ 18,4\% DM1 (8,3\% DM2). Es zeigte sich eine signifikant erhöhte Prävalenz von „Rechtschreibproblemen“ (Chi-Quadrat-Test nach Pearson $\mathrm{p}=0,039$ ), „Schwierigkeiten beim Kopfrechnen“ (Chi-QuadratTest nach Pearson $\mathrm{p}=0,043$ ) und Einstufung der Patienten „mit Lernproblemen“ (Chi-Quadrat-Test nach Pearson $\mathrm{p}=0,012$ ) bei DM1-Patienten. Die Gruppe aus DM1-Patienten mit mindestens einer Auffälligkeit (Antwort: „ja“) in den Bereichen „Lesen lernen“, „Rechtschreibung erlernen“, „Schreiben lernen“ und „Kopfrechnen“ bildet ein Kollektiv aus 62 Patienten (49,6\% des gesamten DM1-Kollektivs). Als höchsten Schulabschluss erreichten von dieser Untergruppe 33,3\% einen Hauptschulabschluss, 24,1\% die mittlere Reife und 38,9\% die (Fach-)Hochschulreife. Im Vergleich zum gesamten, unselektierten DM1-Kollektiv war bei den Prozentwerten zum Hauptschulabschluss $(p=0,080)$ sowie zur (Fach-)Hochschulreife ( $p=0,161)$ ein deutlicher, wenn auch nicht signifikanter Unterschied nachzuweisen.

Berufliche Ausbildung: Bei dieser Untersuchung reduzierte sich das Gesamt-DM-Kollektiv auf 277 Patienten (DM1: $N=122$; DM2: $N=155$ ), da sich vier Patienten noch in Ausbildung befinden. Der Anteil an DM1-Patienten mit (Fach-)Hochschulabschluss (25\%) übersteigt dabei geringfügig denjenigen der DM2-Patienten (23\%). Gegenläufig verhält es sich bei der normalen, beruflichen Ausbildung/Lehre (DM1: 61\%; DM2: 71\%) (• Abb. 2a, b).

\section{Diskussion}

Bereits in den frühen Studien zur Myotonen Dystrophie Typ 1 Curschmann-Steinert wurde eine kognitive Beteiligung diskutiert [5], wenngleich der Schwerpunkt der Erkrankungsbeschreibung zunächst auf den muskulären Symptomen oder somatischen Begleitsymptomen (kardial, endokrin, ophthalmologisch) lag. Patienten mit DM1 weisen eine erhöhte Prävalenz an kognitiver Beeinträchtigung auf, u.a. in Form von Intelligenzminderung, Konzentrationsschwäche, Lese- und Rechtschreibbeeinträchtigung, Autismus-Spektrums-Erkrankungen und Fatigue [13, 16-21]. Für Patienten mit DM1 steht im Alltag die kognitive Komponente oft im Vordergrund, die Ärzte und Therapeuten als eine ernstzunehmende und komplexe Herausforderung ansehen. 
Tab. 1 Allgemeiner Schulabschluss von DM1- und DM2-Patienten (im Vergleich zur Normalbevölkerung [29]).

\begin{tabular}{|c|c|c|c|c|c|c|c|c|c|c|c|c|}
\hline \multirow{6}{*}{$\begin{array}{l}\text { Bildungs- } \\
\text { stand/ } \\
\text { allgemeiner } \\
\text { Schulab- } \\
\text { schluss }\end{array}$} & \multirow{5}{*}{$\begin{array}{l}\text { Durch- } \\
\text { schnitts- } \\
\text { alter }\end{array}$} & \multirow[t]{5}{*}{ Insgesamt } & \multicolumn{10}{|l|}{ Davon } \\
\hline & & & \multirow{4}{*}{\multicolumn{2}{|c|}{$\begin{array}{l}\text { noch in schuli- } \\
\text { scher Ausbildung }\end{array}$}} & \multicolumn{6}{|c|}{ mit allgemeinem Schulabschluss } & \multirow{2}{*}{\multicolumn{2}{|c|}{$\begin{array}{l}\text { ohne allgemei- } \\
\text { nen Schulab- } \\
\text { schluss }\end{array}$}} \\
\hline & & & & & \multicolumn{2}{|l|}{ zusammen } & \multicolumn{4}{|l|}{ Davon } & & \\
\hline & & & & & & & Hauptschul- & Mittlere & (Fach-) & ohne & & \\
\hline & & & & & & & abschluss & Reife & $\begin{array}{l}\text { Hochschul- } \\
\text { reife }\end{array}$ & $\begin{array}{l}\text { Angaben } \\
\text { zur Art } \\
\text { des Ab- } \\
\text { schlusses }\end{array}$ & & \\
\hline & [a] & N & $\mathbf{N}$ & $\%$ & $\mathbf{N}$ & $\%$ & & & & & $\mathbf{N}$ & $\%$ \\
\hline $\begin{array}{l}\text { Bevölkerung } \\
\text { nach allge- } \\
\text { meinem } \\
\text { Schulab- } \\
\text { schluss } 2012\end{array}$ & k.A. & $71421^{*} 10^{3}$ & $2657^{*} 10^{3}$ & 3,7 & $65799 * 10^{3}$ & 92,1 & 38,6 & 31,5 & 29,6 & 0,2 & $2700^{*} 10^{3}$ & 3,8 \\
\hline $\begin{array}{l}\text { Myotone } \\
\text { Dystrophie } \\
\text { Typ } 1\end{array}$ & 43,73 & 125 & 3 & 2,4 & 115 & 92,0 & 20,9 & 26,1 & 50,4 & 2,6 & 7 & 5,6 \\
\hline $\begin{array}{l}\text { Myotone } \\
\text { Dystrophie } \\
\text { Typ } 2\end{array}$ & 54,86 & 156 & 1 & 0,6 & 147 & 94,2 & 17,7 & 31,3 & 48,3 & 2,7 & 8 & 5,1 \\
\hline
\end{tabular}

k.A. = keine Angaben.

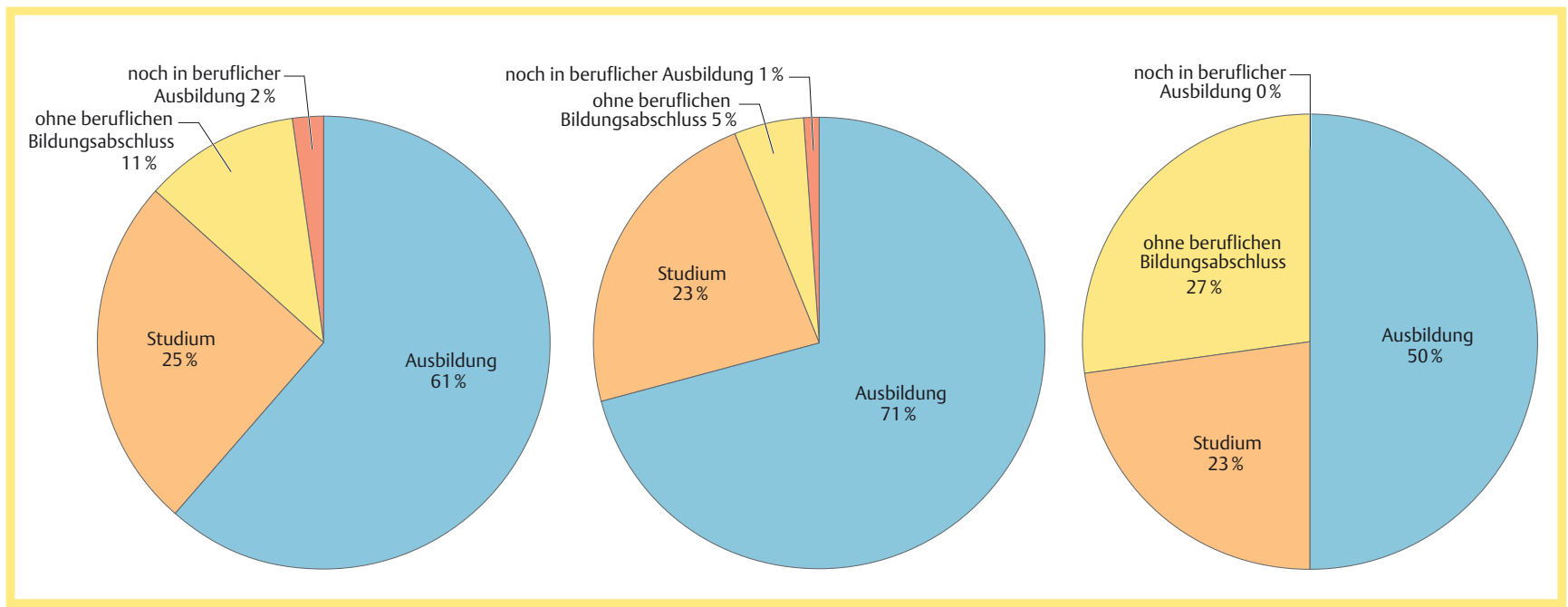

Abb.2 Beruflicher Bildungsstand von DM1-Patienten a und DM2-Patienten b (im Vergleich zur Normalbevölkerung c [30]).

In Langzeitstudien zur juvenilen DM1 konnte gezeigt werden, dass die intellektuellen und kognitiven Beeinträchtigungen parallel zu den muskulären Symptomen progredient verlaufen. Andererseits aber ergaben u.a. repetitive Erhebungen des GesamtIQs bei diesen Patienten, dass der IQ entweder unverändert blieb oder eine nur geringe Abnahme aufwies [22]. cMRT-Untersuchungen zur Korrelation von kognitiven Beeinträchtigungen und strukturellen Veränderungen des Nervensystems lieferten kontroverse Ergebnisse. Während einige Studien eine deutlichere, über das Altersmaß hinausgehende Hirnatrophie mehr bei DM1- als bei DM2-Patienten zeigten [23 - 27], dominierte diese Beobachtung in anderen Untersuchungen bei den DM2-Patienten [28]. Die Zusammenhänge zwischen zerebralen Veränderungen und dem klinischen Phänotyp sind bislang noch nicht ausreichend verstanden und bedürfen weiterer multimodaler Studien.
Insgesamt scheint eher eine neuronale Entwicklungsstörung als ein degenerativ-demenzieller Prozess bei der DM1 vorzuliegen, während bei der DM2 eher neurodegenerative Prozesse im Vordergrund stehen.

Unser Interesse galt der Fragestellung, ob sich in der schulischen und beruflichen Laufbahn Hinweise für eine kognitive Beeinträchtigung nachweisen lassen oder ob ggf. die sehr typische Facies myopathica bei DM1 diese kognitive Beeinträchtigung nur suggeriert und ob sich Unterschiede zu der DM2-Patientengruppe herausstellen.

Vergleicht man das Bildungsniveau von DM1 und DM2, so weichen Patienten mit DM1 und DM2 hinsichtlich des höchsten Schulabschlusses überraschenderweise nicht voneinander ab. In allen schulischen Bereichen (Hauptschulabschluss, mittlere Reife, Fach-/Hochschulreife) besteht eine maximale Differenz 
von $5,2 \%$, die somit keinen signifikanten Unterschied darstellt. Mit einer Differenz von 10\% für Lehre/Ausbildung bzw. 2\% für Fach(hoch)schul-/Hochschulabschluss verhält es sich im Bereich „beruflicher Bildungsabschluss“ gleichermaßen. Diese hohe Ähnlichkeit in den Ergebnissen legt nahe, dass sich beide Patientengruppen hinsichtlich der schulischen und beruflichen Ausbildung deutlich weniger voneinander unterscheiden, als es bislang im klinischen Alltag und in den klinischen Kollektivbeschreibungen angenommen wird [9]. Einschränkend bei dieser Bewertung müssen aber naturgemäß unsere Stichprobengröße und die Nichteinbeziehung der am stärksten betroffenen DM1-Untergruppe berücksichtigt werden. Nicht unerwartet zeigt sich eine Diskrepanz zwischen den beiden DM-Formen bezüglich der Gruppe von Patienten „ohne beruflichen Bildungsabschluss“. Eine fehlende Berufsausbildung kommt bei DM1 mit $11 \%$ mehr als doppelt so häufig vor wie bei DM2 mit $5 \%$. Beim Vergleich der erreichten Schulabschlüsse der DM-Patienten mit der Normalbevölkerung in Deutschland zeigt sich ein überraschend deutlicher Unterschied. 38,6\% der Normalbevölkerung erzielten einen Hauptschulabschluss, während es im DM1-Kollektiv 20,9\% bzw. 17,7 \% im DM2-Kollektiv sind. Im Gegensatz dazu erreichten 50,4\% der DM1- und 48,3\% der DM2-Patienten das Abitur und diese Zahlen liegen damit deutlich höher als die der Abiturabschlüsse der Normalbevölkerung mit 29,6\% [29]. Hinsichtlich der beruflichen Ausbildung/Lehre findet sich eine mehr als 20\% ige Differenz zwischen der Normalbevölkerung und unseren Patientenkollektiven (DM1 61\%, DM2 71\%, Normalbevölkerung $50 \%$ [30]) (o Abb. 2c). Wir sind uns bewusst, dass aus einer möglichen Vorselektion, die aufgrund der verstärkten Teilnahmebereitschaft an einer leistungsbezogenen Umfrage von Patienten mit höherem Bildungsniveau entsteht, ein positiveres Abschneiden unserer Patientenkollektive gegenüber der Normalbevölkerung erklärt werden könnte. Die Ergebnisanalyse unterliegt somit einem gewissen Bias und zeichnet nur ein teilrepräsentatives Bild aller DM-Patienten. Etwaige Einflussfaktoren auf die schulische und berufliche Entwicklung wie eine starke FatigueSymptomatik sowie Konzentrationsschwierigkeiten und eine reduzierte Stresstoleranz, die vor allem bei Patienten mit hohen Repeatexpansionen bekannt sind, wurden anhand einer gesonderten Analyse untersucht. Die Subgruppe - bestehend aus allen DM1-Patienten mit Ausnahme jener mit einer Repeatverlängerung $>900$ - weist dabei keine statistisch signifikanten Unterschiede zu dem gesamten DM1-Kollektiv in den Bereichen schulischer $(p=0,991)$ und beruflicher Abschluss $(p=0,995)$ auf. Aufgrund der niedrigen Fallzahl von 13 DM1-Patienten mit einer CTG-Repeatlänge > 900 schlägt sich der Einfluss neuropsychologischer Defizite nur in unerheblichem Maße in den Ergebnissen nieder. Einen weiteren statistisch signifikanten Unterschied ergab die Auswertung der Lernschwierigkeiten während der Schulzeit. In den Variablen „Rechtschreibung erlernen“ und „Kopfrechnen“ gab ein größerer Anteil der DM1-Patienten als der DM2Patienten $(p=0,039)$ Probleme an. Auch wurden deutlich mehr Patienten des DM1-Kollektivs mit Lernschwierigkeiten eingestuft $(p=0,012)$. Im Gegensatz dazu sind die Angaben der beiden Patientengruppen zu der Frage nach Schwierigkeiten beim „Lesen Lernen“ nahezu identisch (DM1: 17,6\%; DM2: 17,3\%). Die signifikanten Unterschiede zwischen dem DM1- und dem DM2Kollektiv bei den Schwierigkeiten des „Rechtschreibung-Erlernens“ und „Kopfrechnens“ während der Schulzeit sprechen dafür, dass die LRS-/Dyskalkulie-Thematik die beiden DM-Formen voneinander differenziert. Obwohl in der Literatur unterschiedliche Angaben zur Häufigkeit von Lese-/Rechtschreibstörungen und Dyskalkulie zu finden sind, liegen die Prozentwerte für die Normalbevölkerung stets im einstelligen Bereich und damit deutlich unter den Ergebnissen dieser Studie [31]. Ursachen für die oben beschriebenen Unterschiede der Häufigkeit von Lese-/ Rechtschreibstörungen und Dyskalkulie sind weitgehend unklar; sie könnten auf einer eher neuronalen Entwicklungsstörung bei DM1 versus eine neurodegenerativen Komponente bei DM2 beruhen [2]. Eingehendere Untersuchungen dieser speziellen Untergruppe der DM-Patienten mit Lese-/Rechtschreibstörungen und Dyskalkulie unter Einbeziehung auch eines standardisierten Intelligenztests sind in Arbeit und werden versuchen zur weiteren Klärung beizutragen. Es könnte sich daraus für die schwerer betroffenen DM1-Patienten eine neue langfristige Therapieoption ergeben.

\section{Take Home Message}

Patienten mit Myotonen Dystrophien weisen überzufällig häufig eine kognitive Einschränkung auf, die jedoch in dem untersuchten Patientenkollektiv keine Auswirkung auf die schulische und berufliche Ausbildung hat. Unterschiede zwischen DM1- und DM2-Patienten für den höchsten Schulabschluss sind statistisch nicht signifikant. Vielmehr scheint das Vorliegen einer Lese-/Rechtschreibschwäche bzw. Dyskalkulie bei DM1-Patienten für die weitere kognitive und soziale Entwicklung ein kennzeichnender Diskriminator zu sein, weshalb für ein zukünftig besseres Verständnis der Kognition der Myotonen Dystrophien neben formalen IQ-Tests die Untersuchung eines großen DM-Patientenkollektivs mittels standardisierter Lese-Rechtschreibtests sinnvoll und notwendig werden wird. Eine frühzeitige Abklärung des kognitiven Status sollte sich im medizinischen Alltag etablieren, um eine für den Patienten individuell angepasste Therapie, wie Lese- oder kognitive Verhaltenstherapie, zu ermöglichen [14]. Neben einer verbesserten Lebensqualität und Selbstständigkeit kann dadurch die berufliche Ausbildung gefördert und das soziale Umfeld gesichert und entlastet werden.

\section{Danksagung \\ $\nabla$}

Die Autoren danken allen teilnehmenden Patienten herzlich. Inhalte dieser Arbeit sind Teil einer medizinischen Dissertationsarbeit von Frau K. Stahl.

Wir danken Herrn Marzahn und der Diagnosegruppe Myotone Dystrophien der Deutschen Gesellschaft für Muskelkranke e.V. für die finanzielle Unterstützung dieser Pilotstudie.

Interessenkonflikt: Die Autoren geben an, dass kein Interessenkonflikt besteht.

\section{Literatur}

1 Hirschfeld G, Berneburg M, von Arnim C et al. Progeroid syndromes: clinical symptoms and molecular causes of premature aging. Deutsches Ärzteblatt-Köln 2007; 104: 292

2 Mateos-Aierdi AJ, Goicoechea M, Aiastui A et al. Muscle wasting in myotonic dystrophies: a model of premature aging. Front Aging Neurosci 2015; 7

3 Steinert $H$. Über das klinische und anatomische Bild des Muskelschwunds der Myotoniker. Dtsch Z Nervenheilkd 1909; 37: 58 - 104

4 Adie WJ. Dystrophia myotonica (myotonica atrophica). Brain 1923; 46: $73-127$ 
5 Woodward JB 3rd, Heaton RK, Simon DB et al. Neuropsychological findings in myotonic dystrophy. J Clin Neuropsychol 1982; 4: 335-342

6 Maas 0 , Paterson AS. Mental changes in families affected by Dystrophia Myotonica. The Lancet 1937; 229: 21 -23. DOI: http://dx.doi.org/ 10.1016/S0140-6736(00)86562-9

7 Perini GI, Menegazzo E, Ermani $M$ et al. Cognitive impairment and (CTG)n expansion in myotonic dystrophy patients. Biol Psychiatry 1999; 46: 425-431

8 Liquori CL, Ricker K, Moseley ML et al. Myotonic dystrophy type 2 caused by a CCTG expansion in intron 1 of ZNF9. Science 2001; 293: 864 867. DOI: $10.1126 /$ science.1062125

9 Day JW, Ricker K, Jacobsen JF et al. Myotonic dystrophy type 2: molecular, diagnostic and clinical spectrum. Neurology 2003; 60: 657-664

10 Meola G. Clinical aspects, molecular pathomechanisms and management of myotonic dystrophies. Acta Myol 2013; 32: 154-165

11 Harper PS, Harley HG, Reardon $W$ et al. Anticipation in myotonic dystrophy: new light on an old problem. Am J Hum Genet 1992; 51: 10 16

12 Phillips MF, Steer HM, Soldan JR et al. Daytime somnolence in myotonic dystrophy. J Neurol 1999; 246: 275-282

13 Heatwole C, Bode R, Johnson $N$ et al. Patient-reported impact of symptoms in myotonic dystrophy type 1 (PRISM-1). Neurology 2012; 79: 348 - 357. DOI: 10.1212/WNL.0b013e318260cbe6

14 van Engelen B. Cognitive behaviour therapy plus aerobic exercise training to increase activity in patients with myotonic dystrophy type 1 (DM1) compared to usual care (OPTIMISTIC): study protocol for randomised controlled trial. Trials 2015; 16: 224. DOI: 10.1186/s13063-0150737-7

15 Disability-Team. Dyslexia questionnaire. Liverpool: Liverpool John Moores University; 2015

16 Steyaert J, de Die-Smulders C, Fryns JP et al. Behavioral phenotype in childhood type of dystrophia myotonica. Am J Med Genet 2000; 96: 888-889. DOI: 10.1002/1096-8628(20001204)96:6<888::AIDAJMG42>3.0.CO;2-7 [pii]

17 Douniol M, Jacquette A, Guile JM et al. Psychiatric and cognitive phenotype in children and adolescents with myotonic dystrophy. Eur Child Adolesc Psychiatry 2009; 18: 705-715. DOI: 10.1007/s00787-0090037-4

18 Cohen D, Plaza $M$, Angeard $N$ et al. Reading and spelling impairments in children and adolescents with infantile myotonic dystrophy. Journal of Neurolinguistics 2006; 19: 455 -465. DOI: http://dx.doi.org/10.1016/j. jneuroling.2006.03.007

19 Douniol M, Jacquette A, Cohen D et al. Psychiatric and cognitive phenotype of childhood myotonic dystrophy type 1 . Dev Med Child Neurol 2012; 54: 905 -911. DOI: 10.1111/j.1469-8749.2012.04379.x
20 Jean S, Richer L, Laberge $L$ et al. Comparisons of intellectual capacities between mild and classic adult-onset phenotypes of myotonic dystrophy type 1 (DM1). Orphanet J Rare Dis 2014; 9: 186. DOI: 10.1186/ s13023-014-0186-5

21 Ekstrom AB, Hakenas-Plate L, Samuelsson L et al. Autism spectrum conditions in myotonic dystrophy type 1 : a study on 57 individuals with congenital and childhood forms. Am J Med Genet B Neuropsychiatr Genet 2008; 147B: 918 -926. DOI: 10.1002/ajmg.b.30698

22 Echenne B, Rideau A, Roubertie A et al. Myotonic dystrophy type I in childhood Long-term evolution in patients surviving the neonatal period. Eur J Paediatr Neurol 2008; 12: 210-223. DOI: 10.1016/j. ejpn.2007.07.014

23 Kassubek J, Juengling FD, Hoffmann S et al. Quantification of brain atrophy in patients with myotonic dystrophy and proximal myotonic myopathy: a controlled 3-dimensional magnetic resonance imaging study. Neurosci Lett 2003; 348: 73 -76. DOI: S0304394003007407 [pii]

24 Weber YG, Roebling R, Kassubek $J$ et al. Comparative analysis of brain structure, metabolism, and cognition in myotonic dystrophy 1 and 2. Neurology 2010; 74: 1108-1117. DOI: 10.1212/ WNL.0b013e3181d8c35f

25 Franc DT, Muetzel RL, Robinson PR et al. Cerebral and muscle MRI abnormalities in myotonic dystrophy. Neuromuscul Disord 2012; 22: 483 - 491. DOI: 10.1016/j.nmd.2012.01.003

26 Schneider-Gold C, Bellenberg B, Prehn C et al. Cortical and Subcortical Grey and White Matter Atrophy in Myotonic Dystrophies Type 1 and 2 Is Associated with Cognitive Impairment, Depression and Daytime Sleepiness. PLoS One 2015; 10: e0130352. DOI: 10.1371/journal.pone.0130352

27 Minnerop M, Weber B, Schoene-Bake JC et al. The brain in myotonic dystrophy 1 and 2: evidence for a predominant white matter disease. Brain 2011; 134: 3530 - 3546. DOI: 10.1093/brain/awr299

28 Kornblum C, Reul J, Kress $W$ et al. Cranial magnetic resonance imaging in genetically proven myotonic dystrophy type 1 and 2. J Neurol 2004; 251: 710 - 714. DOI: 10.1007/s00415-004-0408-1

29 Statistisches-Bundesamt. Statistisches Jahrbuch 2014. 78. Wiesbaden: Statistisches Bundesamt (Hrsg); 2014

30 Statistisches-Bundesamt. Statistisches Jahrbuch 2014. 80. Wiesbaden: Statistisches Bundesamt (Hrsg); 2014

31 Roeske D, Ludwig KU, Neuhoff $N$ et al. First genome-wide association scan on neurophysiological endophenotypes points to trans-regulation effects on SLC2A3 in dyslexic children. Mol Psychiatry 2011; 16: 97 - 107. DOI: $10.1038 / \mathrm{mp} .2009 .102$ 